\title{
Themen
}

Bernd Hagenau

\section{Von Weinnestern und goldenen Söhnen}

\author{
Aus den Anfangsjahren der Arbeitsgemeinschaft der \\ Regionalbibliotheken
}

The early years of the Working Group of Regional Libraries

http://doi.org/10.1515/bd-2019-0050

Zusammenfassung: Nach dem Zweiten Weltkrieg waren die Bemühungen um den Auf- und Ausbau des deutschen Bibliothekswesens vornehmlich auf die Universitätsbibliotheken gerichtet, während die Landesbibliotheken als Stätten des historischen Erbes ihrer jeweiligen Regionen an den Rand gedrängt zu werden drohten. Ihr maßgeblich von Dr. Hermann Sauter (Landesbibliothek Speyer) initiierter Zusammenschluss zur Arbeitsgemeinschaft der Landesbibliotheken im Jahre 1958 verschaffte ihnen bibliothekspolitisches Gewicht: 1964 fanden sie Einlass in die „Empfehlungen des Wissenschaftsrates zum Ausbau der wissenschaftlichen Einrichtungen“. Durch die Vereinigung mit der Arbeitsgemeinschaft der kommunalen wissenschaftlichen Bibliotheken im Jahre 1971 entstand die Arbeitsgemeinschaft der Regionalbibliotheken. Der vorliegende Beitrag blickt zurück insbesondere auf die Anfänge dieser sechzigjährigen Geschichte, skizziert aber auch in groben Zügen deren weiteren Verlauf. Er wurde in verkürzter Form auf der Herbsttagung der Arbeitsgemeinschaft der Regionalbibliotheken als Festvortrag präsentiert.

Schlüsselwörter: Bibliotheksgeschichte 20. Jahrhundert, Bibliothekspolitik 20. Jahrhundert, Hermann Sauter (1907-1985), Regionalbibliotheken, Landesbibliotheken

Abstract: After the Second World War, efforts to develop and expand the German library system mainly focused on academic university libraries. Regional state libraries were at risk of being pushed to the margins despite their pivotal role in maintaining the cultural and historic heritage of their region. Due to Dr. Hermann Sauter's (of the Speyer regional library) initiating the association of the working

Bernd Hagenau: b.hagenau@sulb.uni-saarland.de 
group of regional libraries in 1958, they gradually regained relevance in library policies. In 1964 regional libraries were included in the "recommendations of the Council of Science and Humanities for the development of scientific institutions“. By joining forces with the working group of municipal scientific libraries in 1971, the Standing Working Group of Regional Libraries was finally established. Our contribution looks back to the beginnings of the sixty-year history and outlines its developments in broad terms. A shorter version was presented as keynote lecture at the autumn meeting of the Working Group of Regional Libraries.

Keywords: library history and library policies in the 20th century, Hermann Sauter (1907-1985), regional libraries, state libraries

\section{Einleitung}

Es ist fürwahr sehr ehrenvoll, um einen Festvortrag ${ }^{1}$ anlässlich des sechzigjährigen Bestehens der Arbeitsgemeinschaft der Regionalbibliotheken gebeten zu werden, darf man sich schließlich in eine Reihe stellen mit hochgeschätzten früheren Kollegen wie Gerhard Römer oder Paul Raabe, denen die Würdigung vergangener runder Geburtstage der Arbeitsgemeinschaft oblag. Die Ehre ist zugleich zwiespältig, denn üblicherweise wird für derartige Zwecke eine Person angefragt, die auch für den Vorsitz im Ältestenrat einer solchen Gruppierung in Frage käme.

Nun muss ich freimütig gestehen, dass ich der Gründung unserer Arbeitsgemeinschaft in meinem zweiten Lebensmonat, dem Wonnemonat Mai des Jahres 1958, nicht die Aufmerksamkeit gewidmet habe, die ihr fraglos gebührt hätte. Jene Anfänge der Arbeitsgemeinschaft haben meine beiden geschätzten Vorredner bereits hinlänglich aufgearbeitet, ${ }^{2}$ wobei sie auf einen entsprechenden Beitrag von Wolfgang Dittrich ${ }^{3}$ zurückgreifen konnten. Die in dessen Aufsatz

1 Die vorliegende Fassung des Festvortrages zum sechzigjährigen Bestehen der Arbeitsgemeinschaft der Regionalbibliotheken, gehalten am 8. November 2018 in Fulda, wurde für die Drucklegung leicht überarbeitet.

2 Römer, Gerhard: Vielfalt als Problem und Chance. Überlegungen zum 40jährigen Bestehen der Arbeitsgemeinschaft der Regionalbibliotheken im Deutschen Bibliotheksverband, Sektion 4. In: DBV-Jahrbuch 1998. Berlin 1999, S. 162-173. Der Festvortrag von Paul Raabe zum fünfzigjährigen Bestehen, gehalten am 29.09.2008 in Halle, ist leider nicht veröffentlicht worden.

3 Dittrich, Wolfgang: Zur Geschichte der Arbeitsgemeinschaft der Regionalbibliotheken in der Sektion 4 des Deutschen Bibliotheksverbandes. In: Ruppelt, Georg (Hg.): Bibliothekspolitik in Ost und West. Geschichte und Gegenwart des Deutschen Bibliotheksverbandes. Frankfurt a. M. 1998 (ZfBB Sonderheft 72, S. 170-177). 
enthaltenen einschlägigen Literaturhinweise sind ergänzt, aktualisiert und konzentriert in einer jüngeren Publikation von Irmgard Siebert zu finden, die freilich über die historische Betrachtung hinaus eher eine grundlegende Blickrichtung verfolgt. ${ }^{4}$ Den Ausgangspunkt für die historische Betrachtung der Arbeitsgemeinschaft der Regionalbibliotheken bildet jedoch ein Beitrag von Wilhelm Totok aus dem Jahre 1975, der freilich damals bereits die Sinnfälligkeit einer solchen Rückschau in Frage gestellt, zugleich aber den größten Erfolg der Regionalbibliotheken und ihres Zusammenschlusses gewürdigt hat, nämlich der Gefahr entronnen zu sein, „sowohl von den Unterhaltsträgern als auch von der bibliothekarischen Fachwelt [...] in eine Rolle hineingezwungen zu werden, in der sie nur noch eine große Vergangenheit, aber kaum mehr eine Zukunft hatten. “5

Die eigenständige Profilierung von Regionalbibliotheken war stets ein wichtiges Anliegen, durchaus in Abgrenzung zu den anscheinend allgegenwärtigen und alles dominierenden Universitätsbibliotheken. Diese Einschätzung hat sich seither freilich geändert, nicht zuletzt, weil inzwischen auch die Daseinsberechtigung von Universitätsbibliotheken keine Selbstverständlichkeit mehr ist. Wir werden darauf zurückkommen.

\section{Quellenlage}

Die Quellenlage zur Geschichte der Arbeitsgemeinschaft der Landes- und später der Regionalbibliotheken ist in Teilen durchaus spärlich, selbst ihre Protokolle sind nur in wenigen Häusern annähernd vollständig erhalten. Mein Dank gilt daher Frau Dr. Marianne Riethmüller, unserer heutigen Gastgeberin, die mir auf eine entsprechende Anfrage per Rundmail hin kurzerhand Scans der in Fulda vorhandenen Protokolle bzw. Tätigkeitsberichte der Arbeitsgemeinschaft aus den 1960er Jahren zukommen ließ. Damit dürfte sich in Fulda, immerhin auch dem Gründungsort der Arbeitsgemeinschaft, vermutlich die vollständigste Sammlung solcher Unterlagen befinden. Mit dem Jahr 1968 beginnt dann auch die Sammlung

\footnotetext{
4 Siebert, Irmgard: Die Zukunft liegt in der Vergangenheit. Historische Bibliotheken auf dem Weg zu Forschungsbibliotheken. In: Bibliothek, Forschung und Praxis 37 (2013), S. 78-90.

5 Totok, Wilhelm: Von den Anfängen der Arbeitsgemeinschaft der Landesbibliotheken; in: Bieber, Hedwig; Kutscher, Siegfried; Wehefritz, Valentin (Hg.): Stadtbibliothek und Regionalbibliographie. Festschrift für Hans Moritz Meyer. Berlin 1975, S. 15-26, hier: S. 15: „Welchen Nutzen hat, so könnte man fragen, heute, wo uns so viele Gegenwarts- und Zukunftsprobleme auf den Nägeln brennen, der rückwärts gerichtete Blick auf die Anfänge einer Arbeitsgemeinschaft von Bibliotheken (...)?“
} 
der Protokolle in der Württembergischen Landesbibliothek, die bis etwa 2009 meiner Einschätzung nach - weitgehend vollständig sein dürfte. Die Kolleginnen und Kollegen in Stuttgart haben mir die Einsichtnahme in diese Sammlung ermöglicht, nachdem ich diesbezüglich noch mit unserem geschätzten Kollegen Dr. Hannsjörg Kowark in Kontakt gestanden habe, dem ich nunmehr, wie Sie alle erfahren mussten, nur noch posthum für seine Unterstützung danken kann.

Vom Hörensagen war mir bekannt, dass sich in der Gottfried Wilhelm Leibniz Bibliothek in Hannover weitere Dokumente befinden sollten, die Herr Dr. Wolfgang Dittrich während seiner Amtszeit als Vorsitzender zwischen 1989 und 1996 gesammelt hatte. Gerade zur rechten Zeit erreichte mich ein Hinweis unserer dortigen Kollegin Frau Anne May, dass im Rahmen einer akut anzuberaumenden Umräumaktion auch zwei ältere Aktenordner mit Unterlagen der fünfziger und sechziger Jahre aufgetaucht seien. Diese erwiesen sich für mich dann in der Tat als überaus beachtenswert, und manche Erkenntnislücke ließ sich dank ihrer Hilfe schließen.

Ich darf Sie dennoch gleich vorab insofern beruhigen, als die Geschichte der Arbeitsgemeinschaft nicht neu geschrieben zu werden braucht. Es ist auch offenkundig, dass meinen Vorrednern die jetzt in Hannover befindlichen Unterlagen ebenfalls vorgelegen haben dürften, vielleicht in anderer Fassung oder Vollständigkeit. Insbesondere Wilhelm Totok zeichnet den - beim Aktenstudium nicht immer so deutlich erkennbaren - roten Faden der frühen Jahre recht detailliert nach.

Eine künftige weiterführende Betrachtung könnte sich möglicherweise auf Unterlagen in den Archiven anderer Gründungsmitglieder der Arbeitsgemeinschaft richten, die in ihrer Gesamtheit gewiss eine annähernde Vollständigkeit aufweisen werden. Auch der Nachlass Hermann Sauters in der Pfälzischen Landesbibliothek zu Speyer wäre gewiss eine eingehende Sichtung wert, die für den vorliegenden Beitrag nicht vorgenommen werden konnte.

\section{Die Situation der Regionalbibliotheken in der Nachkriegszeit}

In den 1950er Jahren oblag der Wiederaufbau und Ausbau der deutschen Wissenschaft zunächst der Notgemeinschaft der Deutschen Wissenschaft als Vorgängerin der heutigen Deutschen Forschungsgemeinschaft. Diese hatte ihre Bibliotheksförderung jedoch - nicht zuletzt in Folge des sogenannten „Scheibertschen Gutachtens“ - auf Universitätsbibliotheken konzentriert, mit der Württembergischen Landesbibliothek als einziger Ausnahme. Den Landesbibliotheken attes- 
tierte jener Geschichtsprofessor Peter Scheibert, sie dienten nicht in erster Linie der Forschung, sondern eher der Landesgeschichte oder Allgemeinbildung. ${ }^{6}$

Sie standen also im Abseits der Wissenschaftsentwicklung der Nachkriegszeit, und ihre historisch begründete Diversität tat ein Übriges, um die eine oder andere von ihnen im Fortbestand zu gefährden. Zudem drohten sie angesichts der Neugründung von Hochschulen und des Ausbaus öffentlicher Stadtbibliotheken zwischen alle Stühle zu geraten. Und so ist es dem wahrhaft unermüdlichen Eifer des Direktors der Pfälzischen Landesbibliothek in Speyer, Dr. Hermann Sauter, zu verdanken, dass er die Initiative zur Gründung der Arbeitsgemeinschaft der Landesbibliotheken ergriff, um diesem Bibliothekstypus eine politische Lobby zu verschaffen.

Drei Jahre später, im August 1961, konnte Sauter in ihrem Namen ein Memorandum an den Wissenschaftsrat versenden, das die Bedeutung der Landesbibliotheken an der wissenschaftlichen Literaturversorgung untermauerte. Dass diese 1964 Eingang gefunden haben in die „Empfehlungen des Wissenschaftsrates zum Ausbau der wissenschaftlichen Einrichtungen, Teil II: Wissenschaftliche Bibliotheken“, 7 dürfte wohl bis heute als größter Erfolg der Arbeitsgemeinschaft gelten. Ausgiebig und minutiös für jede einzelne Einrichtung sind dort Empfehlungen für deren Ausbau mit Personal- und Sachmitteln aufgenommen worden. ${ }^{8}$ Eingangs schon wird deren grundsätzliche Bedeutung für ein ausgebautes Bibliotheksnetz in der Bundesrepublik Deutschland gewürdigt, insbesondere im Hinblick auf die schnell fortschreitende Industrialisierung und „Verwissenschaftlichung“ zahlreicher Lebensbereiche. Deshalb seien auch außerhalb der Hochschulen bibliothekarische Schwerpunkte „in genügender Anzahl und nicht zu großer Entfernung voneinander"9 erforderlich. Auch im späteren Bibliotheksplan '73 fanden sich die Regionalbibliotheken als Einrichtungen der 3. Stufe wieder. ${ }^{10}$ Ihr Beitrag im Netz der überregionalen Literaturversorgung hatte damit eine nachhaltige Anerkennung erfahren. Dessen Neubearbeitung zwanzig Jahre später stellt die Regionalbibliotheken in der Funktionsstufe 3 an die Seite der Hochschulbibliotheken und widmet ihnen einen eigenen Unterabschnitt. ${ }^{11}$

6 Vgl. Römer, wie Anm. 1, insbes. S. 163-164.

7 Tübingen 1964.

8 Ebenda, S. 76-143.

9 Ebenda, S. 24.

10 Bibliotheksplan '73. Entwurf eines umfassenden Bibliotheksnetzes für die Bundesrepublik Deutschland. Berlin 1973.

11 Bibliotheken '93. Strukturen, Aufgaben, Positionen. Berlin/Göttingen 1994, S. 38-41. 
Einen weiteren Meilenstein im Wirken dieser Arbeitsgemeinschaft bildete 1971 die Veröffentlichung des Sammelbandes „,Regionalbibliotheken in Deutschland“, ${ }^{12}$ gemeinsam herausgegeben mit der Arbeitsgemeinschaft der Kommunalen Wissenschaftlichen Bibliotheken, mit der sich die Arbeitsgemeinschaft der Landesbibliotheken noch im selben Jahr auf dem Kölner Bibliothekartag unter dem Dach des Vereins Deutscher Bibliothekare zur Arbeitsgemeinschaft der Regionalbibliotheken zusammengeschlossen hatte. Dieser erfuhr im Jahre 2000 eine Neubearbeitung. ${ }^{13}$

Wenn ich bereits den unermüdlichen Eifer Hermann Sauters hervorgehoben habe, so erweist sich diese Formulierung beim Studium der Akten eher als eine Untertreibung. Auch im vorelektronischen Zeitalter verging oftmals kaum ein Tag zwischen dem Erhalt eines Schreibens und einer Gegenantwort, keine Gelegenheit durfte ungenutzt verstreichen, die Anliegen der Landesbibliotheken an jeder mehr oder weniger geeigneten Stelle zu artikulieren.

\section{Die Gründung der Arbeitsgemeinschaft der Landesbibliotheken (1958)}

Im hannoverschen Aktenmaterial findet sich ein Schreiben des Direktors der Niedersächsischen Landesbibliothek, Dr. Gerhard Meyer, vom 13. Mai 1958, der eine Antwort auf einen - dort nicht erhaltenen - Brief Sauters vom Vortag darstellt, „den ich“, wie Meyer schreibt, „sofort beantworten will“. Gemeinsam mit seinem bereits konsultierten Kollegen Dr. Wolfgang Fischer von der Landesbibliothek Oldenburg unterstützt er darin Sauters Vorschlag, im Rahmen des anstehenden Bibliothekartages in Fulda ein Treffen der Direktoren der Landesbibliotheken einzuberufen: „Die Mittagszeit des Mittwoch scheint mir sehr passend, wir haben dann bis drei Uhr Zeit. Ich würde Herrn Pieper an Ihrer Stelle für ein Lokal sorgen lassen, wo wir ungestört uns vereinigen können. Ich wünsche Ihnen vollen Erfolg. Sie werden die Sache ja mit gewohnter Energie in Angriff nehmen.“

12 Totok, Wilhelm; Weimann, Karl-Heinz (Hg.): Regionalbibliotheken in der Bundesrepublik Deutschland. Im Auftrag der Arbeitsgemeinschaft der Landesbibliotheken und der Kommunalen Wissenschaftlichen Bibliotheken. Frankfurt a. M. 1971. (Zeitschrift für Bibliothekswesen und Bibliographie, Sonderheft 11).

13 Hagenau, Bernd (Hg.): Regionalbibliotheken in Deutschland. Mit einem Ausblick auf Österreich und die Schweiz. Frankfurt a. M. 2000. (Zeitschrift für Bibliothekswesen und Bibliographie, Sonderheft 78). 
Und ob! ... Sauter hat also seinen Plan zunächst mit einigen ihm näherstehenden Kollegen abgestimmt und nach positiver Rückmeldung dann am 16. Mai 1958 die eigentliche Einladung verschickt. Diese beginnt mit der Anrede: „Sehr geehrter Herr Kollege!“ Sie passte damals auf alle Adressaten. Und der Einladungstext im Wortlaut:

„Nachdem im Vorjahre begonnene Gespräche über Fragen, die alle Landesbibliotheken gemeinsam berühren, der grossen Entfernungen wegen auf schriftlichem Wege nicht weiter gekommen sind, erlaube ich mir Sie, zugleich im Namen von Herrn Kollegen Dr. Meyer (Hannover) und Herrn Kollegen Dr. Fischer (Oldenburg) einzuladen, an einer gemeinsamen Besprechung in Fulda teilzunehmen.

Da das Programm der Tagung sowieso schon stark besetzt ist, scheint uns die Mittagszeit am Mittwoch, den 28.5. als einzige bzw. beste Gelegenheit zu einer Aussprache zu bleiben. Ich möchte vorschlagen, dass wir uns nach dem Vortrag von Herrn Kollegen Pieper beim Mittagessen zusammensetzen und anschließend - bis zum Beginn der Nachmittagsvorträge - über die Fragen aussprechen, die uns gemeinsam beschäftigen. Als wichtige Punkte dieser Besprechung dürften sich die Fragen der Einstufung der Direktoren der Landesbibliotheken und die eines Mindest- bzw. Normaletats von Landesbibliotheken - je nach Größenordnung - ergeben.

Falls Sie noch irgend einen Vorschlag für die Besprechung machen möchten, darf ich um Ihre möglichst baldige Äußerung bitten.

Ich würde mich aufrichtig freuen, wenn Sie sich unserem gemeinsamen Bemühen nicht versagen wollten.

Mit kollegialen Grüssen

Ihr sehr ergebener“

plus Unterschrift. Man beachte so ganz nebenbei, dass ein Bibliothekartag damals seinen festen Programmablauf hatte und nicht auf etliche parallele Themenkreise, Workshops, Präsentationen, Newcomer Foren und dergleichen mehr Rücksicht genommen werden musste. Jeder wusste, wann was wo stattfindet und entsprechend auch, wann die lieben Kollegen Zeit für andere Dinge hatten.

Über der erhaltenen Durchschrift dieses Einladungsschreibens ist mit Schreibmaschine im Original hinzugefügt worden: „Stuttgart, Karlsruhe, Wiesbaden, Fulda, Oldenburg, Hannover, Düsseldorf, Dortmund, Kiel, Kassel, Coburg, Detmold, Darmstadt.“ Damit - zuzüglich Speyer als Absender des Briefes dürften die Adressaten für das Mittagessen und somit die potenziellen Gründungsmitglieder der Arbeitsgemeinschaft der Landesbibliotheken genannt sein.

Es trudelten die Antworten ein, wenige sind auch erhalten, so etwa eine mit 10 Pfennig frankierte Postkarte mit einer spröden Zusage aus Karlsruhe und einer etwas komplizierteren brieflichen Absage aus Coburg vom 20. Mai 1958: Der dortige Direktor, Dr. Friedrich Knorr, sei in den Bundestag gewählt worden und 
daher aus dem Bibliotheksdienst ausgeschieden. Der neue Leiter der Bibliothek, Herr Bibliotheksrat Dr. Franz Georg Kaltwasser, kein Geringerer als der spätere Generaldirektor der Bayerischen Staatsbibliothek also, sei urlaubsbedingt ebenfalls verhindert. Und so bittet der Verfasser des Schreibens, Bibliotheksoberinspektor Ehrfried Baumgärtel, freundlich darum, „etwa gefasste Beschlüsse der Landesbibliothek Coburg mitzuteilen“.

Dieser höfliche Schlusssatz aus Coburg wurde von Hermann Sauter gewissenhaft beachtet, und so verdanken wir ihm ein Schreiben vom 12. Juni 1958, in dem Baumgärtel darüber unterrichtet wird, ,daß die in Fulda vertretenen Landesbibliotheken sich zu einer Arbeitsgemeinschaft zusammengeschlossen haben, zu deren Vorsitzenden man den Unterzeichneten [sic] gewählt hat“, verbunden mit der herzlichen Einladung an die Bibliothek in Coburg, „unserer Arbeitsgemeinschaft beizutreten.“

Wir können damit also vom 28. Mai 1958 als dem Gründungstag der Arbeitsgemeinschaft der Landesbibliotheken ausgehen, denn Hinweise auf eine kurzfristige Terminverschiebung finden sich nicht, auch wenn Pieper eine solche angeregt hatte, weil noch kurzfristig ein zusätzlicher Vortrag (Dr. Gisela von Busse) ins Programm aufgenommen wurde und jene Mittagspause am Mittwoch verkürzte.

\section{Die Arbeitsgemeinschaft unter ihrem ersten Vorsitzenden Hermann Sauter (1958-1962)}

Gleich im Juli 1958 beginnt die Arbeit dieser neuen Gemeinschaft, und das allererste Thema, mit dem sie sich in Gestalt ihres rührigen Vorsitzenden beschäftigt, ist durchaus handfester Natur, denn es geht um die Besoldung der Direktoren von Landesbibliotheken.

Im Mittelpunkt steht dabei die Einstufung des Kollegen Dr. Franz Pieper in Fulda, der sich noch in der Besoldungsgruppe A 13 befindet, während man eigentlich schon dabei ist, die Überführung der Direktoren wissenschaftlicher Bibliotheken von der Besoldungsgruppe A 14 nach A $15 \mathrm{zu}$ betreiben, was bis zu diesem Zeitpunkt offenbar nur in Baden-Württemberg erfolgt war, dort übrigens in Landes- wie Universitätsbibliotheken gleichermaßen. $\mathrm{Zu}$ jener Zeit wurden Oberstudienräte in Gymnasien gerade in die Besoldungsgruppe A 14 überführt, deshalb hielt man für Bibliotheksdirektoren eine Heraushebung in die A 15 für angemessen. Es folgt ein recht umfänglicher, teilweise ziemlich detailverliebter Briefwechsel mit dem zuständigen Vertreter des Hessischen Ministeriums für Erziehung und Volksbildung, Professor Sante, der in einem Schreiben vom 14. Juli 1958 aufgefordert wird, den ,in schematischen Vorstellungen denkenden 
Ministerialverwaltungen“ energisch entgegen zu treten und sich für die Bibliotheksdirektoren einzusetzen.

Dann aber geht es an die umfängliche Bestandsaufnahme der Landesbibliotheken, um deren Bedeutung für die wissenschaftliche Literaturversorgung in Deutschland zu untermauern. Leihverkehrsfragen rücken in den Blickpunkt, doch schnell zeigt sich, hier insbesondere am Beispiel der Herzog August Bibliothek in Wolfenbüttel, dass solche Zahlen aufgrund der Verschiedenartigkeit der Bibliotheken nur schwer vergleichbar und begrenzt aussagefähig sind. Auch der Versuch, einen „Normaletat“ festzulegen, den eine durchschnittliche Landesbibliothek benötige, scheitert an dem Umstand, dass es eine solche idealtypisch nirgendwo gibt und die finanzielle Ausstattung ohnehin recht unterschiedlich ist. So kommt wenig Unterstützung aus Kiel, wo man mit dem Etat eigentlich ganz gut zurecht komme, ${ }^{14}$ zumal man sich ohnehin eher als eine Spezialbibliothek zum Thema „Schleswig-Holstein“ sehe, ohne den Anspruch, eine breit aufgestellte wissenschaftliche Bibliothek für die Öffentlichkeit sein zu wollen. Schließlich gebe es in Kiel ja auch noch eine Universitätsbibliothek, anders als in Speyer. ${ }^{15}$

Aus Coburg wiederum kommt Unterstützung. Kaltwasser schreibt am 3. November 1958, man solle „keinen Versuch zur Aufbesserung unserer Finanzlage ausser acht lassen“. Und er beantwortet die Leihverkehrsumfrage: Im Rechnungsjahr 1957/58 hat die Landesbibliothek Coburg 42 eigene Werke an fremde Bibliotheken ausgeliehen, davon 22 an Staats- und Hochschulbibliotheken. Ob diese Zahlen die DFG oder den Wissenschaftsrat zu beeindrucken vermochten? ... Gegenüber dem Wissenschaftsrat jedenfalls war zu dokumentieren, dass die Landesbibliotheken „unter Zugrundelegung strenger Maßstäbe“ wissenschaftliche Arbeit leisten. Daher wurden die Anzahl von Studierenden und Wissenschaftlern unter den Nutzern erhoben, dazu Leihverkehrszahlen, Veröffentlichungslisten und Angaben zum Schriftentausch.

Der Wissenschaftsrat war damals gerade erst gegründet worden, sein Aufgabenprofil noch nicht endgültig umrissen. Auf ihm ruhten im Vergleich zur Deutschen Forschungsgemeinschaft die größeren Hoffnungen. Der Wissenschaftsrat erschien außerdem leichter ansprechbar insofern, als die in ihm versammelten Ländervertreter für die Direktoren auf dem Dienstweg problemlos zu erreichen waren. Dennoch klopfte man auch bei der DFG an, jedoch ohne Erfolg. Auf einen entsprechend negativen Beschluss der DFG im Jahre 1959 reagiert Wolfgang Fischer aus Oldenburg recht ungehalten gegenüber Sauter, ${ }^{16}$ spricht von

14 Schreiben vom 30. Oktober 1958.

15 Schreiben vom 5. November 1958.

16 Schreiben vom 19. September 1959. 
einem erwartbaren Ergebnis der bekannten „Cliquenwirtschaft“ in der DFG und ihrem engeren Umfeld, das er - etwas kryptisch - als „Berlins goldene Söhne“ bezeichnet. Haben wir hier schon eine Vorwegnahme dessen, was der Konstanzer Bibliotheksdirektor Dr. Joachim Stoltzenburg genau dreißig Jahre später als den ,inneren Kreis“ bezeichnen sollte? ${ }^{17}$ Sauter selbst reagiert in seiner Antwort vom 22. Oktober 1959 weitaus diplomatischer: Er habe bei der DFG in Bonn ein „freundliches Klima“ vorgefunden, Dr. Kurt Zierold, der Generalsekretär der DFG, erschien ihm ,viel zu intelligent, als dass er die sachliche Berechtigung des grundsätzlichen Anspruchs der Landesbibliotheken nicht klar erkennt, und auch zu anständig, als dass er eine solche Kenntnis einer unsachlichen Intrige“ opferte. Immerhin lehnte die DFG nicht die Förderung einzelner Projekte ab, jedoch eine „Beteiligung an zentralen Mitteln“. Damit dürfte ein regelmäßiger Zuschuss zum Erwerbungsetat gemeint gewesen sein.

Doch noch einmal zurück zur Landesbibliothek Coburg: Konnte Sauter die Information, dass deren vorheriger Leiter ${ }^{18}$ nun im Bundestag saß, gänzlich unbeeindruckt lassen? Natürlich nicht.

Es entspinnt sich auch zwischen Sauter und Knorr ein Briefwechsel, allerdings mit längeren Pausen, der aus heutiger Sicht einen gewissen Unterhaltungswert besitzt. Eine erste Annäherung Sauters scheitert unter Hinweis auf zahlreiche Terminverpflichtungen des Abgeordneten sowie auf den bayerischen Wahlkampf. Dann folgt Knorrs relativ lockere Zusage, sich bei Bundesinnenminister Dr. Gerhard Schröder für die Landesbibliotheken einsetzen zu wollen. Das fortwährende Scheitern einer gemeinsamen Terminfindung zwischen Sauter und Knorr liest sich in den Akten fast schon als running gag. Manchmal ist Sauter verhindert, einen der angebotenen Termine wahrzunehmen, meistens aber muss der vielbeschäftigte Politiker passen, der Sauter am 4. Juli 1960 schreibt: „Sollten Sie auch einmal in östlicher Richtung oder nach München fahren, dann könnten wir uns eventuell in Würzburg oder in einem der umliegenden Weinnester vorher treffen. Wir müssen ja zu unserer Besprechung nicht unbedingt in meinem Büro in Bonn sein.“ Danach verebbt dieser Dialog, zu einem Treffen scheint es nie gekommen zu sein.

17 Stoltzenburg, Joachim: Der Innere Kreis als Zentrum deutscher Bibliothekspolitik - Strukturen und Prozeduren. In: Bibliotheksdienst 23 (1989), S. 481-498.

18 Dr. Friedrich Knorr (1904-1978) leitete die Landesbibliothek Coburg von 1949 bis zu seiner Wahl in den Deutschen Bundestag 1957 (vgl. Habermann, Alexandra; Klemmt, Rainer; Siefkes, Frauke: Lexikon deutscher wissenschaftlicher Bibliothekare 1925-1980. Frankfurt a. M. 1985, Zeitschrift für Bibliothekswesen und Bibliographie, Sonderheft 24, S. 163). Seine dortige Aufbauarbeit wurde in Fachkreisen hoch gelobt, vgl. seinen Nachruf in: Bibliotheksforum Bayern 6 (1978), S. 161. 
Schon 1958 standen Überlegungen im Raum, ob nicht auch die - wissenschaftlichen - Stadtbibliotheken in die Initiative einbezogen werden sollten. Sauter sprach sich dagegen aus, man wollte sich fokussieren und nicht verzetteln. Im folgenden Jahr taucht die Frage auf, ob sich die Arbeitsgemeinschaft dem Verein Deutscher Bibliothekare als Kommission andienen sollte. Damals war der VDB keine reine Personenvertretung, sondern leistete in großem Umfang auch bibliothekarische Sacharbeit. Oder wäre es nicht besser, als Arbeitskreis mit beratender Funktion für politische Entscheidungsträger zu fungieren? ${ }^{19}$ Leider können diese Diskussionen anhand des Aktenmaterials nicht immer in aller Ausführlichkeit nachverfolgt werden, denn bisweilen sind auch nur Versatzstücke solcher Korrespondenzen erhalten.

Dr. Ludwig Denecke, der neue Direktor der Murhardschen Bibliothek der Stadt Kassel und Landesbibliothek, wird ab $1960 \mathrm{zu}$ einem der bevorzugten Gesprächspartner Hermann Sauters. Denecke unterstützt die Einbeziehung der Stadtbibliotheken, die sich bereits mit dem Gedanken tragen, eine eigene Arbeitsgemeinschaft zu gründen: „Eine Zusammenfassung der Nicht-Universitätsbibliotheken hat doch die stärksten gemeinsamen Interessen und scheint mir als geschlossene Gemeinschaft eine zweckmäßigere Vertretung als in einer Aufteilung.“20 Eingangs des Schreibens verweist er unvermittelt auf eine Vorgängerinstitution, auf die auch Gerhard Römer in seinem Festvortrag von 1998 hingewiesen hat: „Mir sagte Kollege Hopf, daß er selbst die Arbeitsgemeinschaft ,Landes- und Stadtbibliotheken' ins Leben gerufen habe.“

Sauter scheint dies auch nicht gewusst zu haben, denn er fragt seinerseits bei Fischer in Oldenburg nach, ob der etwas über diese Vorgängerinstitution wisse. ${ }^{21}$ Offenbar nicht. Und direkt als Antwort an Denecke am 22. Januar 1960: „Sehr wichtig ist mir zu erfahren, dass schon einmal eine Arbeitsgemeinschaft Landesund Stadtbibliotheken bestanden hat. Könnte ich darüber Näheres erfahren? Kollege Hopf steht nicht im Jahrbuch, so dass ich nicht weiß, wohin ich eine Anfrage an ihn richten soll.“ Da Dr. Wilhelm Hopf, ${ }^{22}$ einer seiner Amtsvorgänger in Kassel, allerdings erkrankt ist, tut sich auch Denecke schwer damit, weitere Auskünfte einzuholen. Sauter hakt am 24. März 1960 nach: „Es wäre doch sehr schön und nützlich, wenn man über die Fragen Arbeitsgemeinschaft der Landesund Stadtbibliotheken bald etwas erfahren könnte, möglichst vielleicht auch so

19 Schreiben von Fischer an Sauter, 9. Mai 1959.

20 Schreiben vom 16. Januar 1960.

21 Schreiben vom 22. Januar 1960.

22 Dr. Wilhelm Hopf (27.07.1876-21.09.1962) war von 1920 bis 1938 und dann wieder von 1944 bis 1949 Direktor der Landesbibliothek Kassel; vgl. Habermann; Klemmt; Siefkes: Lexikon deutscher wissenschaftlicher Bibliothekare 1925-1980, wie Anm. 18, S. 135-136. 
rechtzeitig, dass man die Kollegen von den in Betracht kommenden Stadtbibliotheken schon zu einer gemeinsamen Besprechung nach Trier bitten könnte." In Trier fand der Bibliothekartag 1960 statt, auf dem die Arbeitsgemeinschaft, wie schon im Vorjahr in Freiburg, eine eigene Sitzung abhielt.

Erst am 3. Mai meldet sich Denecke in dieser Angelegenheit zurück: „Als ich jetzt Herr Kollege [sic] Hopf einmal wieder besuchen konnte, erhielt ich von ihm die beifolgenden Blätter, die Ihnen Aufschluß über die Gründung der alten Arbeitsgemeinschaft der Landes- und Stadtbibliotheken geben. Ich hoffe, daß Ihnen damit gedient ist.“

In den hannoverschen Akten finden sich danach in der Tat zwei Blätter, wobei das zweite jedoch lediglich (damals) aktuelle Bestandszahlen einiger Landesbibliotheken enthält und offenbar nicht zu den beiden erwähnten „Hopfschen“ Blättern gehört. Auf dem ersten Blatt hingegen steht zu lesen:

„Bei der Sitzung der Arbeitsgemeinschaft der Landes- und Stadtbibliotheken im Jahre 1933 in Darmstadt waren folgende Landes-, Staats- und Stadtbibliotheken vertreten:

Bremen, SB

Darmstadt, LB

Dortmund, LB

Hannover, StB

Hannover, K V u. Pr. B. [sic]

Karlsruhe, LB

Kassel, LB

Oldenburg, LB

Speyer, LB

Stuttgart, LB

Wiesbaden, LB

Wolfenbüttel, Herzogl. B.

[hier folgen zwei Leerzeilen]

Dessau, LB

Dresden, LB

Weimar, LB

Auf einer Liste von 1930 waren als Teilnehmer einer Gründungsversammlung noch genannt worden folgende Bibliotheken:

Düsseldorf, LuStB

Fulda, LB

Kiel, LB 
Lübeck, StB

Mainz, StB

[Leerzeile]

Ratibor, LB

Schwerin, LB“

Die Leerzeilen machen deutlich, dass die ostdeutschen Bibliotheken in eine eigene alphabetische Reihenfolge gebracht wurden. ${ }^{23}$

Mehr ist über diese Vorgängerinstitution anhand der hannoverschen Akten leider nicht in Erfahrung zu bringen. ${ }^{24}$ Das „Zentralblatt für Bibliothekswesen“ berichtet in seinen Jahrgängen 1930 und 1933 ausführlich über die jeweiligen Bibliothekartage in Lübeck ${ }^{25}$ bzw. Darmstadt, ${ }^{26}$ doch ist von der genannten Arbeitsgemeinschaft nirgends die Rede. Beim leider eher in anderer Hinsicht denkwürdigen Bibliothekartag 1933 in Darmstadt haben sich aber zumindest zwei Beiträge mit Landesbibliotheken befasst, nämlich der Gastgeber Hans Wilhelm Eppelsheimer höchstselbst ${ }^{27}$ mit einem eher grundsätzlichen Referat „Die Landesbibliothek als Bibliothekstyp“ sowie Joseph Theele, der Fuldaer Bibliotheksdirektor, mit seiner Kurzdarstellung „Der Neubau der Landesbibliothek Fulda““ ${ }^{28}$ Hopf wird im Rahmen der Berichterstattung über den Bibliothekartag lediglich abschließend als derjenige erwähnt, der „besondere Veranlassung“ empfunden habe, dem „Vorstande für die Vorbereitung und Durchführung der Tagung zu danken, besonders dem Vorsitzenden“. In Klammern und kursiv folgt der Zusatz: „(Starker Beifall!) ‘29

23 Gerhard Römer muss ein ähnliches Dokument vorgelegen haben, auf dem die Sortierung in jeweils zwei Alphabete noch als Desiderat vermerkt war. Vgl. Anm. 2, S. 162. Von den an die Mitglieder versandten Dokumenten der Arbeitsgemeinschaft existieren neben dem Original möglicherweise sogar unterschiedliche Kopien bzw. Ausfertigungen. Sicherlich dürfte sich auch irgendwo das von Denecke erwähnte zweite Blatt (evtl. noch weitere, denn er spricht ja nur von „beifolgenden Blättern“) auffinden lassen, das in Hannover offenbar fehlt oder vertauscht wurde. 24 Auch in einem Schriftenverzeichnis von Wilhelm Hopf sind keine Beiträge über eine solche Arbeitsgemeinschaft zu finden, wie Hopf überhaupt nur sehr wenige bibliothekarische Artikel verfasst hat, dafür umso mehr bemerkenswerte Aufsätze zur hessischen Geschichte; vgl. Brauns, Eduard: Wilhelm Hopf. Verzeichnis seiner Schriften. In: Zeitschrift des Vereins für hessische Geschichte und Landeskunde 63 (1952), S. 128-134. Die letzten Einträge stammen aus dem Jahre 1951. In den letzten elf Jahren seines Lebens scheint Hopf damit nicht mehr als Autor in Erscheinung getreten zu sein. Möglicherweise aus gesundheitlichen Gründen?

25 Zentralblatt für Bibliothekswesen 47 (1930), S. 377 ff.

26 Zentralblatt für Bibliothekswesen 50 (1933), S. $501 \mathrm{ff}$.

27 Zentralblatt für Bibliothekswesen 50 (1933), S. 525-528.

28 Ebenda, S. 587-589.

29 Ebenda, S. 596. 
Erkennbar ist jedoch der Versuch, die Arbeitsgemeinschaft zu vergrößern, und zwar zunächst um weitere Landesbibliotheken im engeren Sinne, wenngleich nicht mit dieser Titulierung, nämlich die Staatsbibliotheken in Bremen und Bamberg sowie die Herzog August Bibliothek Wolfenbüttel. Diese werden 1960 explizit als Mitglieder angefragt, wobei eine Antwort aus Bamberg nicht zu finden ist. Am 21. November 1960 ergeht eine neuerliche Umfrage zum Thema „Normetat" an folgende Bibliotheken: Bremen, Coburg, Darmstadt, Detmold, Dortmund, Düsseldorf, Fulda, Hannover, Karlsruhe, Kassel, Kiel, Oldenburg, Stuttgart, Wiesbaden, Wolfenbüttel. Sind dies neben Speyer die Mitglieder der Arbeitsgemeinschaft Ende 1960? Dann wäre Bamberg trotz Aufforderung in der Tat nicht dabei gewesen, die Gruppe insgesamt aber auf 16 Mitglieder angewachsen.

Durch ein ansonsten eher zusammenhanglos erhaltenes Schreiben vom 4. Januar 1962 Dr. Paul Geisslers von der Staats- und Stadtbibliothek Augsburg an die durch Dr. Jürgen Busch (Mainz) repräsentierte Arbeitsgemeinschaft kommunaler wissenschaftlicher Bibliotheken erfahren wir nun auch von deren Existenz. ${ }^{30}$ Es finden sich weitere Schreiben in den Akten, die auf Buschs Aktivitäten zur Vergrößerung dieser neuen Arbeitsgemeinschaft hindeuten, die auf dem Bibliothekartag 1961 in München gegründet worden zu sein scheint. ${ }^{31}$ Ein Schreiben Buschs an den Direktor der Stadtbibliothek Hannover, Dr. Rolf Kluth, vom 1. September 1961 listet die angefragten Bibliotheken auf, wobei sich durchaus eine Schnittmenge mit der Zielgruppe der Arbeitsgemeinschaft der Landesbibliotheken ergab: Aachen, Augsburg, Berlin, Bochum, Bonn, Braunschweig, Dortmund (StB u. LB), Düsseldorf (LB u. StB), Essen, Hanau, Hannover, Hildesheim, Kassel (LB), Lübeck, Lüneburg, Mainz, Mannheim (StB), Mönchen-Gladbach, München, Nürnberg, Schweinfurt, Trier, Ulm, Worms, Wuppertal, Konstanz. Noch nicht

30 Über die Entstehung und Anfangszeiten der Arbeitsgemeinschaft kommunaler wissenschaftlicher Bibliotheken sind wir nur bruchstückhaft informiert, es fehlt eine zusammenhängende Darstellung. Vielleicht ist dieses Informationsdefizit auch dem frühen Tod ihres Gründers Jürgen Busch geschuldet, der bereits am 21. März 1965 mit nur 40 Jahren verstarb (vgl. Habermann, Klemmt, Siefers: Lexikon deutscher wissenschaftlicher Bibliothekare 1925-1980, wie Anm. 18, S. 43).

31 So auch Gerhard Römer, vgl. Anm. 1, S. 166. In einer tabellarischen Darstellung der Geschichte der AG Regionalbibliotheken nennt Ludger Syré jedoch 1963 als Gründungsjahr der AG der Kommunalen Wissenschaftlichen Bibliotheken: https:/www.bibliotheksverband.de/fachgruppen/ arbeitsgruppen/regionalbibliotheken/ueber-uns/geschichte.html [Zugriff: 21.02.2019]. Geisslers Schreiben vom 04.01.1962 stärkt jedoch die Annahme einer Gründung bereits 1961. Eine zwei Jahre spätere Gründung wäre allerdings plausibel, wenn es sich bei Buschs Anfragen eher um informelle Vorabfragen der Bereitschaft zur Mitwirkung an einer solchen noch zu gründenden AG handelte. 
angeschrieben, aber im Blickfeld waren: Duisburg, Gelsenkirchen, Bielefeld, Mülheim, Saarbrücken.

In den Unterlagen gehen die Aktivitäten der beiden Arbeitsgemeinschaften nun immer häufiger durcheinander oder auch ineinander über. Der Direktor der Stadtbibliothek Nürnberg, es dürfte Dr. Karlheinz Goldmann gewesen sein, ${ }^{32}$ artikuliert am 18. September 1961 seine Zweifel, ob die Arbeitsgemeinschaft der Stadtbibliotheken sich mehr den wissenschaftlichen Stadtbibliotheken oder den „Einheitsbibliotheken“ zuwenden werde, womit die öffentlichen Bibliotheken im Sinne der „Public Library“ gemeint gewesen sein dürften. Auch diese Arbeitsgemeinschaft überhäuft ihre Mitglieder mit Fragebögen, wobei vor allem Vorarbeiten für das erste KGSt-Gutachten ${ }^{33}$ in den Blickpunkt rücken, während sich die Arbeitsgemeinschaft der Landesbibliotheken nach ihrem erfolgreichen Vorstoß beim Wissenschaftsrat wieder vermehrt Besoldungsfragen zuwendet.

\section{Die Arbeitsgemeinschaft unter ihrem zweiten Vorsitzenden Wilhelm Totok (1962-1970)}

Sauter hat für die Bibliothekartage mindestens seit 1960 alljährlich einen Bericht über seine Tätigkeit als Vorsitzender der Arbeitsgemeinschaft verfasst. Darüber hinaus deutet nichts auf weitere Zusammenkünfte der Arbeitsgemeinschaft hin. Zum 1. April 1962 wechselt Sauter nach Mainz und übernimmt die Leitung der Universitätsbibliothek. Angesichts seines vorherigen beherzten Eintretens für die Belange der Landesbibliotheken gegen deren „Bedrohung“ durch die Universitätsbibliotheken ein erstaunlicher, für ihn persönlich vermutlich aber vorwärts gerichteter Schritt. Die Landesbibliothek Speyer leitet er in seinen ersten Mainzer Monaten sogar noch nebenamtlich weiter und übergibt den Vorsitz der Arbeitsgemeinschaft an seinen hannoverschen Kollegen Dr. Wilhelm Totok, nicht ohne vorher auch auf dem Bibliothekartag 1962 in Darmstadt noch pflichtgemäß über seine Tätigkeit im vorangegangenen Jahr berichtet zu haben.

Über Totoks Anfänge in dieser Funktion wissen wir nichts, denn erst zwei Jahre später findet sich die nächste Spur aus diesen Anfangszeiten der Arbeitsgemeinschaft, nun erstmals in Gestalt eines „Protokolls“, so auch überschrieben und fünf Tagesordnungspunkte aufweisend, von der gemeinsamen Sitzung der

32 Vgl. Habermann, Klemmt, Siefers: Lexikon deutscher wissenschaftlicher Bibliothekare 19251980, wie Anm. 18, S. 96-97.

33 Kommunale öffentliche Bücherei. Kommunale Gemeinschaftsstelle für Verwaltungsvereinfachung. Köln 1964. 
beiden Arbeitsgemeinschaften der Landes- bzw. Stadtbibliotheken am 21. Mai 1964 in Kassel, 15 bis 18 Uhr, somit wiederum auf einem Bibliothekartag. Bemerkenswert ist dabei ein Beitrag der Repräsentantin der Deutschen Forschungsgemeinschaft, Dr. Gisela von Busse. Sie „regt die Erstellung einer Modell-Liste von in- und ausländischen Zeitschriften an“, was schon zu Sauters Zeiten ohne großen Erfolg versucht wurde. Auch ein „Modell-Etat für die Landesbibliotheken und Stadtbibliotheken“ wird wieder einmal erörtert, doch „gehen die Meinungen auseinander“. Nichts Neues also gegenüber den Anfangszeiten unter Sauter, wie überhaupt die bibliothekarischen Themen einschlägiger Versammlungen über die Jahrzehnte hinweg eine erstaunliche Konstanz aufzuweisen vermögen. ${ }^{34}$

Das Protokoll ist von Totok unterzeichnet, im Briefkopf wird, neben der Niedersächsischen Landesbibliothek, nur die AG Landesbibliotheken genannt. Auch in den Folgejahren wurden „Niederschriften“, so die bevorzugte Bezeichnung, der gemeinsamen Sitzungen auf den Bibliothekartagen angefertigt, 1965 in Nürnberg, 1966 in Hannover. Aus der zunächst mitunter so bezeichneten AG der Stadtbibliotheken ist inzwischen offiziell die AG der Kommunalen Wissenschaftlichen Bibliotheken geworden. 1967 in Aachen allerdings tagte die Arbeitsgemeinschaft der Landesbibliotheken für sich allein, zunächst jedenfalls, erst in der „letzten halben Stunde nahmen auch die Vertreter der Arbeitsgemeinschaft der Kommunalen Wissenschaftlichen Bibliotheken an der Sitzung teil“. Bedenkt man, dass auch hier die DFG vertreten war, jetzt durch Dr. Horst Braun, verdeutlicht dies zugleich die Bandbreite, wenn nicht gar den Spagat zwischen Forschungsnähe und Grenzbereich zum öffentlichen Bibliothekswesen. 1968 in Karlsruhe wiederum tagten beide Arbeitsgemeinschaften gemeinsam, und diesmal wird auch der gemeinsame Vorsitz durch Totok und Dr. Hans Moritz Meyer von der Stadtbibliothek Dortmund erwähnt, die beide als Unterzeichner des Protokolls (wie es jetzt auch im Titel heißt) angegeben sind. Die Anzahl der Teilnehmer lag bei 35, ähnlich wie schon in den Vorjahren außer 1967, als die Landesbibliotheken zunächst allein tagten und die Teilnehmerzahl deshalb bei nur 13 lag. 1969 beim Bibliothekartag in Kiel wiederum blieben die Landesbibliotheken unter sich, anwesend waren „21 Kolleginnen und Kollegen aus 16 Bibliotheken“. Nun also auch ein paar Kolleginnen. Eine Namensliste existiert jedoch nicht. Auch 1970 in Augsburg tagten die Landesbibliotheken allein, Totok legte den Vorsitz nieder, was er schon ein Jahr zuvor angekündigt hatte, und übergab ihn an seinen

34 Vgl. Hagenau, Bernd: Gehabte Sorgen hab“ ich gern? Ein Blick zurück auf die Deutschen Bibliothekartage 1975 bis 1980. In: Harms, Ilse; Luckhardt, Heinz-Dirk; Giessen, Hans W. (Hg.): Information und Sprache. Beiträge zu Informationswissenschaft, Computerlinguistik, Bibliothekswesen und verwandten Fächern. Festschrift für Harald H. Zimmermann. München 2006, S. 219-227. 
Wolfenbütteler Kollegen Dr. Paul Raabe. Dieser vertritt die Arbeitsgemeinschaft nun auch in der Satzungskommission des geplanten „Bibliothekenverbandes“, dessen Gründung aus Sicht der Landesbibliotheken ebenfalls zugestimmt wird. ${ }^{35}$

\section{Die Arbeitsgemeinschaft unter ihrem dritten Vorsitzenden Paul Raabe (1970-1974)}

Am 8. und 9. März 1971 kommt es anscheinend erstmals zu einer Tagung außerhalb von Bibliothekartagen, und zwar wieder einmal in Fulda. Beide Arbeitsgemeinschaften tagten gemeinsam, es werden jedoch nur 18 Teilnehmer gezählt. Das immerhin zweitägige Treffen sollte als Vorbereitung dienen auf die Sitzung beim Bibliothekartag in Köln und behandelte gemeinsame Probleme von Landesbibliotheken und wissenschaftlichen Stadtbibliotheken.

Das Treffen scheint erfolgreich gewesen $\mathrm{zu}$ sein, denn auf dem anschließenden Bibliothekartag in Köln kommt es am 3. Juni 1971 zur Vereinigung der beiden Arbeitsgemeinschaften, laut Protokoll auf Vorschlag Wilhelm Totoks: „Die beiden Arbeitsgemeinschaften haben in den letzten Jahren immer gemeinsam getagt, und die Teilnehmer versprechen sich von dieser erweiterten Arbeitsgemeinschaft die Möglichkeit zur Intensivierung und Konzentrierung ihrer gemeinsamen Arbeit und eine Stärkung ihrer Position im Gesamtgefüge des deutschen Bibliothekswesens.“ Den Vorsitz dieser neuen Arbeitsgemeinschaft übernimmt wiederum Paul Raabe, Hans Moritz Meyer (Dortmund) wird sein Stellvertreter.

Gleichzeitig wird die Arbeitsgruppe Regionalbibliographie gegründet, ausgehend von einem Vortrag von Dr. Reinhard Oberschelp (Niedersächsische Lan-

35 Es handelt sich hier um den Deutschen Bibliotheksverband, der letztlich am 13. Juni 1973 auf dem Bibliothekartag in Hamburg gegründet werden sollte als Nachfolger des Deutschen Büchereiverbandes, der seit dem 23. Februar 1949 existierte, jedoch ohne Mitwirkung der wissenschaftlichen Bibliotheken. Noch kurz vor der Gründung des DBV sollte es Ende März bei der Tagung der AG Regionalbibliotheken zu lebhaften Diskussionen über die Satzung des neuen Verbandes kommen. Die Regionalbibliotheken fanden sich darin nicht wieder, weil die Kommissionen, die innerhalb des DBV die Sacharbeit leisten sollten, nicht namentlich genannt waren. 1975 wurde eine erneute Umfrage durchgeführt über die Frage, ob die Arbeitsgemeinschaft als ein Organ des DBV weitergeführt werden sollte: 40 von 50 Bibliotheken antworteten, alle waren dafür, zwei mit Vorbehalten. Problematisch erschien in diesem Zusammenhang auch die Nichtmitgliedschaft Bayerns und Hessens im DBV. Bis 1985 fehlen daher jegliche Hinweise in den Unterlagen, dass es tatsächlich zu einer Einigung mit dem DBV gekommen wäre. Vielmehr dürfte die AG beim VDB verblieben sein. 
desbibliothek Hannover), der das Konzept der landesgeschichtlichen Bibliographie für überholt erklärt. Es sei durch eine Regionalbibliographie zu ersetzen, die als Auswahlkriterium nur die Zugehörigkeit eines Titels zu einem regionalen Problem kenne. Allerdings ist anschließend von dieser Arbeitsgruppe nichts mehr zu hören, vielmehr erfolgt zwölf Jahre später die Ankündigung ihrer ersten (!) Sitzung vom 19. bis 21. Januar 1983. Den Vorsitz dieser Arbeitsgruppe übernimmt von 1984 bis 1988 Horst Lüders (Kiel), seither Dr. Ludger Syré (Karlsruhe). Ihre Tagungen standen anfangs oftmals in einem zeitlichen Zusammenhang mit denen der AG Regionalbibliotheken.

Schon im Herbst 1971 kam es zu einer erneuten Zusammenkunft, damit also der dritten in jenem Jahr, diesmal in Wolfenbüttel und bereits unter dem neuen Namen „Arbeitsgemeinschaft der Regionalbibliotheken“. Die Veranstaltung dauerte vom 27. bis 29. September, wobei ausgiebige Besichtigungen und Rundgänge, ein gemeinsames Mittagessen und ein Empfang im Wohnhaus des Direktors zum Programmablauf gehörten. Dieses mitunter kulturtouristisch anmutende Element sollte fortan, mindestens für den Rest des 20. Jahrhunderts, ein prägendes Merkmal der Arbeitsgemeinschaft der Regionalbibliotheken bleiben und machte aus meiner ganz subjektiven Sicht den besonderen Reiz dieser Reisen an teilweise recht entlegene Orte aus, der seither leider ein wenig verloren gegangen zu sein scheint ...

Die Arbeitsgemeinschaft hatte damals 52 Mitgliedsbibliotheken, darunter inzwischen auch Universitätsbibliotheken mit Landesbibliotheksfunktion. Neben den üblichen Verdächtigen sind im Protokoll beispielsweise genannt: die Stadtbibliotheken Aachen, Braunschweig, Essen, Hannover, Hildesheim, Koblenz, Mönchengladbach, Ulm, Worms und Wuppertal-Elberfeld, die Staatliche Bibliothek Aschaffenburg, die Fürstlich Fürstenbergische Bibliothek Donaueschingen, die Staats- und Seminarbibliothek Eichstätt, die Ratsbücherei Lüneburg, die Fürstlich Thurn und Taxissche Hofbibliothek Regensburg und die Fürstlich Hohenzollernsche Hofbibliothek Sigmaringen. Doch nur 17 der 52 Bibliotheken waren in Wolfenbüttel vertreten. Laut Protokoll bedauert Raabe eingangs der Tagung, „daß eine Reihe von Kollegen aus Mangel an Mitteln für Reisekosten an der Teilnahme verhindert sind.“

Immerhin gelangt man auch zu einer Definition der Regionalbibliotheken anhand von drei Kriterien, die im Protokoll festgehalten sind:

1. Sie verfügen über vorwiegend wissenschaftliche Bestände zur Literaturversorgung außerhalb der Hochschulen.

2. Sie sind zentrale Bibliotheken einzelner Regionen, das können Länder, Gebiete oder Städte sein.

3. Sie sind Stätten der Information, Forschung und Fortbildung. 
Paul Raabe betont die besondere Bedeutung historischer Altbestände, die auch als Abgrenzung gegenüber zahlreichen Universitätsbibliotheken taugen, an deren Aufschwung man dennoch gerne partizipieren würde, und insbesondere Dr. Jürgen Eyssen von der Stadtbibliothek Hannover wiederum hebt die Unterschiede zu öffentlichen Stadtbibliotheken hervor. Von beiden Seiten lauert die Gefahr einer unerwünschten Integration ...

Die Teilnehmerzahlen an Bibliothekartagen, bei denen oftmals auch öffentliche Sitzungen stattfanden, lagen naturgemäß höher. So waren etwa 1972 in Mannheim auch Vertreter der Universitätsbibliotheken Bielefeld, Clausthal und Stellbosch, dazu von der Stadtbibliothek Maastricht aufgelistet. Das mag dem zufälligen Interesse einzelner Besucher geschuldet gewesen sein. Im März 1974 gab Raabe den Vorsitz der AG an Dr. Josef Bellot (Stadtbibliothek Augsburg) ab und übernahm selbst dessen Stellvertretung. Womöglich entsprach dies einer Absprache, die bereits beim Zusammenschluss der ursprünglich getrennten Arbeitsgemeinschaften getroffen wurde.

\section{Die Arbeitsgemeinschaft unter ihrem vierten Vorsitzenden Josef Bellot (1974-1985)}

Es entspinnt sich nun eine lange Folge von Sitzungen im Rahmen von Bibliothekartagen und außerhalb derselben, die Revue passieren zu lassen letztlich zur Ermüdung führen würde. Die großen politischen Debatten der frühen Jahre hatten sich gelegt, flackerten aber bei verschiedenen Gelegenheiten gerne wieder auf, die zahlreichen Positionspapiere, mit denen das Bibliothekswesen beglückt wird oder sich selbst beglückt, blieben praktisch niemals ohne eine Kommentierung aus Sicht der Regionalbibliotheken. Doch auch die Detailarbeit kam nicht zu kurz. 1973 etwa führte die Besichtigung der neuen Württembergischen Landesbibliothek zu merklicher Verstimmung und einer, wie Raabe im Protokoll erwähnt, „doch recht heftigen Kontroverse“ über die dortigen Vitrinen. Auch der abendliche Empfang durch den Rat der Stadt Stuttgart (!) konnte die Laune allenfalls oberflächlich aufbessern. Selbst ein geborener Diplomat wie der Stuttgarter Direktor Dr. Hans-Peter Geh vermochte nur mühsam die Wogen zu glätten, indem er versicherte, die Belegung der Vitrinen sei erst provisorisch erfolgt und weitere Versuche seien „im Gange“.

Allein in den 1970er Jahren wurden den Tagungsteilnehmern im Rahmen ihrer Sitzungen zahlreiche interessante Besichtigungen angeboten, u. a. die Restaurierungswerkstatt in Wolfenbüttel, das Lippische Landesmuseum in Detmold mit dessen Zehntscheune nebst abendlichem Empfang, das Gutenberg Museum und 
das Schloss in Mainz, das Deutsche Literaturarchiv in Marbach, das Mönchengladbacher Münster, das Brüder Grimm-Museum in Kassel, den dortigen Bärenreiter Verlag, das Badische Landesmuseum in Karlsruhe. Dazu natürlich zahllose Bibliotheken, aber auch Rathäuser, in denen Abendempfänge für die Tagungsteilnehmer ausgerichtet wurden. Kein Wunder also, dass die Vorbereitung dieser Veranstaltungen mit einigem Aufwand verbunden war, und so musste der Vorsitzende Bellot auch einige Male passen und die geplanten Sitzungen absagen, einmal mit einer langen Erklärung, dass ihm das Frankfurter Verkehrsamt keinen geeigneten Zeitpunkt im Frühjahr 1977 benennen konnte, an dem die Stadt wegen anderer Veranstaltungen nicht ohnehin schon überfüllt gewesen wäre, ${ }^{36}$ im Herbst 1979 schlicht aus „dienstlichen und privaten Gründen“, außerdem lägen „keine brennenden Probleme an“, wie Bellot hinzufügt. ${ }^{37}$ Überhaupt fehlen Dokumente zwischen 1979 und 1982. In diesem Zeitraum scheinen gar keine Sitzungen der Arbeitsgemeinschaft stattgefunden zu haben. ${ }^{38}$ Bellots Vorschlag für eine Frühjahrssitzung im April 1982 stößt bei seinem Stuttgarter Kollegen Geh auf wenig Gegenliebe, der eine weitere Zusammenkunft so kurz vor dem Bibliothekartag ,aus finanziellen und sachlichen Gründen nicht für gerechtfertigt“"39 hält. Im Oktober kam es dann aber doch zu einer Herbstsitzung in Wolfenbüttel.

\section{Die Arbeitsgemeinschaft als Teil der Sektion 4 im Deutschen Bibliotheksverband}

Mit Erreichen der Altersgrenze legt Bellot auf dem Trierer Bibliothekartag am 30. Mai 1985 sein Amt als Vorsitzender der AG Regionalbibliotheken nieder und schlägt Dr. Gerhard Römer (Badische Landesbibliothek Karlsruhe) als seinen Nachfolger vor. Dessen Wahl erfolgt einstimmig. Raabe bleibt Stellvertreter. Es kommt zugleich die Frage der Anbindung an den DBV wieder auf, die anlässlich dessen Gründung schon mehrfach im Raum gestanden hatte, aber anscheinend zu keinem Abschluss gelangt war. Die AG scheint weiterhin außerhalb des DBV bestanden zu haben, vermutlich unverändert innerhalb des Vereins Deutscher Bibliothekare, so wie schon in den sechziger Jahren. Römer schlägt nun vor, eine AG Regionalbibliotheken innerhalb der Sektion 4 des DBV zu gründen, also quasi

36 Hinweis im Protokoll der Zusammenkunft auf dem Bibliothekartag in Münster 1976.

37 Schreiben Bellots vom 29. Oktober 1979 in der Protokollsammlung der Württembergischen Landesbibliothek.

38 Auch Ludger Syrés Liste (vgl. Anm. 31) weist hier keine Einträge auf.

39 Schreiben Gehs an Bellot vom 16. März 1982 (Protokollsammlung der WLB Stuttgart). 
eine erneute Gründung der eigentlich schon bestehenden Arbeitsgemeinschaft. Er fragt am 19. August 1985 beim DBV-Vorsitzenden Dr. Helmut Sontag nach, welche Konsequenzen sich daraus ergeben könnten, und erhält mit Datum vom 6. September 1985 die Antwort, es gebe keine organisatorischen Bestimmungen für eine solche Arbeitsgemeinschaft. Die Satzung des DBV gelte analog. Man wähle einen Vorsitzenden, der nach Möglichkeit zugleich in den Vorstand der Sektion gewählt werden sollte, dazu evtl. einen oder zwei Vertreter. Allerdings begrenze die Zahl von drei Positionen im Sektionsvorstand diese Möglichkeit. Es habe sich allerdings in der Vergangenheit „nicht als besonders notwendig“ herausgestellt.

Ansonsten seien die Arbeitsgemeinschaften „relativ frei in ihrer Handhabung“, sie sollten lediglich den Vorstand des DBV und den Sektionsvorsitzenden über ihre Aktivitäten und insbesondere über geplante Verlautbarungen informieren. Das Mitgliederverzeichnis werde auf der DBV-Geschäftsstelle geführt. Allenfalls die Geschäftsordnung einer Sektion könne zu einer engeren Regulierung der Arbeitsgemeinschaft führen, doch gebe es derzeit gar keine Geschäftsordnung für die Sektion 4, und an deren künftiger Gestaltung wären die Regionalbibliotheken als Mitglieder der Sektion dann ja schließlich beteiligt. Daraufhin bestanden keine Bedenken mehr gegen eine Eingliederung in die Sektion 4 des Deutschen Bibliotheksverbandes. Ein entsprechender Beschluss wurde auf der Herbstsitzung des DBV am 3. Oktober 1985 in Köln gefasst.

Unter den zahlreichen nachfolgenden Sitzungen sei die Herbstsitzung vom 6. bis 7. November 1990 in Speyer hervorgehoben, auf der bereits erste Berichte aus Regionalbibliotheken der neuen Bundesländer (Schwerin, Potsdam, Halle, Jena und Gotha) vorgetragen wurden. Es wurde beklagt, dass die ostdeutschen Regionalbibliotheken von allen Förderprogrammen zum Ausbau wissenschaftlicher Bibliotheken in den neuen Bundesländern ausgeschlossen seien, weil diese sich allein auf Hochschulbibliotheken konzentrierten. Womit sich der Kreis zu den Anfangszeiten der AG wieder einmal geschlossen hatte ... Immerhin erfolgte die Integration der ostdeutschen Regionalbibliotheken völlig selbstverständlich und geräuschlos. Schon im folgenden November 1991 tagte die Arbeitsgemeinschaft in Dresden. Ihr Vorsitzender war seit 1989 Dr. Wolfgang Dittrich von der Niedersächsischen Landesbibliothek in Hannover, und er sollte es bis zum Herbst 1996 bleiben. Danach begann der Vorsitz in kürzerer Folge zu wechseln, nämlich alle zwei Jahre.

Ein Musterbeispiel an Konstanz wiederum stellt das Amt des Protokollführers dar, „Schriftführer“ genannt. War es zunächst noch üblich, dass der Vorsitzende selbst einen Bericht über seine Arbeit bzw. später das Protokoll einer Sitzung anfertigte, trat in den 1970er Jahren Barbara Strutz als Protokollführerin des Öfteren in Erscheinung. Als Erwerbungschefin in Wolfenbüttel war sie zugleich 
mehrfach eine geschätzte Co-Autorin von Paul Raabe sowie dessen spätere Bibliographin. Von 1985 bis 2002, also siebzehn Jahre lang, erledigte Dr. Hartmut Harthausen von der Pfälzischen Landesbibliothek Speyer diese durchaus mühevolle Aufgabe, unterbrochen lediglich von der einen oder anderen Abwesenheitsvertretung. Sein Nachfolger für immerhin sieben Jahre, 2004 bis 2011, war Detlev Hellfaier von der Lippischen Landesbibliothek in Detmold, und seitdem verdanken wir Ulrich Hagenah diese umfangreiche Dokumentationsarbeit.

Wir müssen jedoch darauf achten, dass diese Arbeit nicht umsonst geleistet wird, denn sogar die Protokollsammlung der Württembergischen Landesbibliothek endet im Jahre 2009, und ich selbst hätte auch meine Schwierigkeiten, alle späteren Protokolle ausfindig zu machen, seitdem sie in elektronischer Form verschickt werden und in irgendwelchen Ordnern landen, die der Beständigkeit eines Servers ausgeliefert sind, bis ihnen eines Tages der Wechsel eines Mailprogrammes den endgültigen Garaus bereitet. Alles ist allgegenwärtig und womöglich irgendwann doch nicht mehr da. Frühzeitig schon ergriff Wolfgang Dittrich eine wirksame Maßnahme gegen die damals noch gar nicht drohende ,digitale Demenz“, indem er während seiner Amtszeit in der Niedersächsischen Landesbibliothek ein Archiv für die Arbeitsgemeinschaft der Regionalbibliotheken einrichtete, das aber leider bei Weitem nicht vollständig ist. Die seit 1996 eingetretenen zahlreichen Wechsel im Vorsitz haben die lückenlose Dokumentation der Vorgänge rund um die AG ebenso wenig erleichtert wie die „Elektrifizierung“ des Informationsaustauschs. Zumindest sollte die Arbeitsgemeinschaft hier zu einer eindeutigen Regelung finden.

\section{Schlussbemerkung}

Beim Rückblick auf die Fragestellungen, die der Gründung der Arbeitsgemeinschaft zugrunde lagen, können wir heute von einer Erfolgsgeschichte sprechen. Alle Bibliothekstypen haben immer wieder mit wechselnden Krisen und ständigem Rechtfertigungsdruck zu kämpfen. Die Regionalbibliotheken aber haben frühzeitig mit der Schärfung ihrer individuellen Profile begonnen und gerade ihre Alt- und Spezialbestände als Schätze und Alleinstellungsmerkmale erkannt. Daran hat die Arbeitsgemeinschaft mit ihren Vorkämpfern einen erheblichen Anteil gehabt. Wenn wir heute auf die Zukunftsfähigkeit der einzelnen Bibliothekstypen schauen, werden Regionalbibliotheken kaum mit durchschnittlichen Universitätsbibliotheken tauschen wollen, denen solche Merkmale fehlen, deren Lizenzierungsgeschäft auch andere Einrichtungen einer Universität übernehmen könnten, deren Lernräume nicht zwangsläufig in einem Bibliotheksge- 
bäude angesiedelt sein müssen und deren Klientel ohnehin den direkten Zugriff auf elektronische Medien ohne Zwischeninstanzen bevorzugt, wie übrigens die Verlagsseite auch. Universitätsbibliotheken besitzen keine Existenzgarantie mehr, eine ihrer modernsten Weiterentwicklungen auf dem Campus Belval der Universität Luxemburg in Esch-sur-Alzette wurde am 24. September 2018 unter der reichlich faden Bezeichnung „Learning Center“ eingeweiht. Ihnen fehlt die Exklusivität der Regionalbibliotheken. Auf tönernen Füßen haben diese sich seinerzeit gegenseitig Halt verschafft, doch das Konstrukt hielt und wurde zunehmend stabiler. Sauters Anfangserfolg, nämlich die Würdigung von Landesbibliotheken durch den Wissenschaftsrat, war ein großer, aber nicht der einzige und noch lange nicht der letzte Schritt zu ihrer Renaissance.

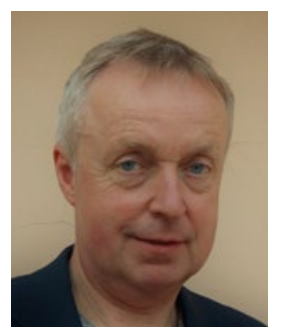

Prof. Dr Bernd Hagenau

Universität des Saarlandes

Campus E1.2

Postfach 11, Zimmer 0.07.1

66123 Saarbrücken

Deutschland

Tel.: +49 (0) 681 302-2510

E-Mail: b.hagenau@sulb.uni-saarland.de 